\section{Case Reports in Ophthalmology}

\title{
Isolated Medial Orbital Wall Fracture Associated with Enophthalmos in a Paediatric Patient: An Unusual Presentation
}

\author{
Panagiotis Giannakouras $^{a} \quad$ Georgios Pollalis $^{b} \quad$ Efthymia Tsina $^{b}$ \\ ${ }^{a}$ Athens University Eye Clinic, G. Gennimatas General Hospital, Athens, Greece; \\ ${ }^{b}$ Department of Ophthalmology, Aghia Sophia Children's Hospital, Athens, Greece
}

\section{Keywords}

Periorbital emphysema $\cdot$ Enophthalmos $\cdot$ Isolated medial orbital wall fracture

\begin{abstract}
Purpose: To report a case of isolated medial orbital wall fracture with enophthalmos in a paediatric patient and describe the clinical presentation and findings by means of computed tomography (CT) of the head and eyes. Methods: We looked at the patient's medical and ophthalmologic history, and an ophthalmologic examination and a CT of the head were performed at baseline. Results: A 14-year-old boy was admitted to the emergency department of our institution with ecchymosis of his right eyelids secondary to a sport accident. Physical examination revealed a moderate limitation of upgaze without diplopia. CT showed a medial orbital wall fracture without haemorrhage and a gross accumulation of air in the right eyelid with pressure exertion over the right globe and enophthalmos. The patient was treated conservatively with oral antibiotics and steroids showing dramatic improvement within 1 week. Enophthalmos and periorbital emphysema were completely resolved within 3 months after the accident as indicated by CT. Conclusions: We conclude that surgical intervention and intravenous treatment are not warranted in similar cases of medial orbital wall fracture. Medical history, clinical and paraclinical evaluations, and a regular follow-up, including CT, are needed though to avoid complications such as painful abduction, horizontal diplopia, pseudo sixth nerve paresis, or pseudo Duane.




\section{Case Reports in Ophthalmology}

Case Rep Ophthalmol 2018;9:126-131

DOI: $10.1159 / 000486275$

C 2018 The Author(s). Published by S. Karger AG, Basel www.karger.com/cop

Giannakouras et al.: Isolated Medial Orbital Wall Fracture Associated with Enophthalmos in a Paediatric Patient: An Unusual Presentation

\section{Introduction}

Herein, we report a case of isolated medial wall fracture after a blunt orbital trauma, this is an unusual type of fracture in children. Young children ( $<13$ years) are more likely to sustain a sport injury or an injury caused by an accident, while older children $(>16$ years) are more prone to be injured during inter-personal violence [1]. A combination of both the hydraulic and force transduction theories is used to explain the pathophysiology of medial wall fractures depending on the mechanism of injury [2].

\section{Case Report}

An otherwise healthy 14-year-old boy was admitted to the emergency unit of the Department of Ophthalmology, Aghia Sophia Children's Hospital, Athens, Greece, complaining of swelling of his right eye. A few hours earlier, he had sustained a blunt trauma to his right orbit as the result of an elbow strike during a basketball game. Physical examination revealed periorbital eyelid swelling with moderate subconjunctival emphysema. No crepitation was present upon palpation. A moderate limitation of upgaze without diplopia was noted (Fig. 1). Visual acuity was $10 / 10$ on the Snellen chart. His pupils were equal in size and normally responsive to light. No paraesthesia of the cheek or teeth was noted. Anterior segment examination and dilated fundoscopy were unremarkable. CT with $2.5 \mathrm{~mm}$ slice thickness showed a minimally displaced fracture of the ethmoid bone without haemorrhage, enophthalmos, and a gross accumulation of air in the right eyelid followed by pressure exertion over the right globe (Fig. 2, 3).

The patient was advised not to blow his nose forcefully. He was treated prophylactically with oral antibiotics and steroids with progressive tapering. The boy was followed up once weekly for a period of 1 month and then every month until 3 months after the accident without any clinical signs or complications (Fig. 1). CT at 1 month showed completely resolved enophthalmos and periorbital emphysema (Fig. 4).

\section{Discussion}

Herein, we report a case of isolated medial wall fracture after a blunt orbital trauma, this is an unusual type of fracture in children. Clinical findings suggestive of a medial wall fracture, in addition to periorbital oedema, ecchymosis, and subconjunctival haemorrhage, which are not pathognomonic, include subcutaneous and subconjunctival emphysema, crepitation, tenderness, epistaxis, and cerebrospinal fluid rhinorrhoea. Damage to the ethmoid bone, causing air to enter between the loose tissues of the orbit after orbital wall fracture, may be presented as periorbital and subconjunctival emphysema [3]. The study by Snell et al. [4] confirms the majority of the literature, suggesting that isolated orbital floor fractures are more common than isolated medial orbital wall fractures, although they may commonly occur together [5]. Lee et al. [6] also noted that medial wall fractures most frequently occur in the anterior aspect of the lamina papyracea, but defect size, rather than position, was a more significant predictor of late enophthalmos. It has been reported that only $10 \%$ of all fractures involving the medial wall occur in isolation [5].

Injuries causing isolated fractures of the medial orbital wall, which are treated conservatively, have been reported to cause enophthalmos in up to $76 \%$ of all patients $[6,7]$. The 
Case Reports in
Ophthalmology

Case Rep Ophthalmol 2018;9:126-131

DOI: $10.1159 / 000486275$

C 2018 The Author(s). Published by S. Karger AG, Basel www.karger.com/cop

Giannakouras et al.: Isolated Medial Orbital Wall Fracture Associated with Enophthalmos in a Paediatric Patient: An Unusual Presentation

main cause of enophthalmos in those cases is a post-traumatic increase in orbital volume [8]. The most comprehensive study of sequelae of medial orbital wall fractures shows that the majority of patients with an isolated medial orbital wall fracture do not present diplopia, enophthalmos, or visual disturbance, making diagnosis on clinical grounds alone difficult [2]. Complications include painful abduction, horizontal diplopia, pseudo sixth nerve paresis, and pseudo Duane [9]. Diplopia may be due to entrapment of the medial rectus muscle [1]. The pathognomonic sign for medial rectus entrapment associated with medial wall fracture is retraction of the globe and narrowing of the palpebral fissure on attempted abduction without any limitation on abduction [10].

Back et al. [11] suggested a conservative approach to facial fractures under the following 3 circumstances: a non-displaced or minimally displaced fracture with minimal symptoms, refusal of treatment, and a patient being medically unfit. Following a conservative treatment, patients are advised to avoid nose blowing, coughing, sneezing, or any activity that may lead to increased pressure in the nasal cavity, including diving and flying by plane [12-15]. Oral antibiotics should be given prophylactically because of the enhanced risk of orbital cellulitis due to microbial spread with forced airflow across the orbital cavity and the sinuses [16]. Steroids may be used to decrease orbital oedema when this is severe [2].

\section{Conclusion}

Our patient represents an unusual case of isolated medial wall fracture in a child that did not involve any orbital emphysema. Our case also confirms that when this type of fracture occurs, it is accompanied by enophthalmos. Our patient met the criteria for conservative treatment, and he did not present any post-traumatic sequelae within 3 months after the accident. This is in accordance with data from the literature pointing out the need for a regular follow-up of those cases.

\section{Acknowledgements}

Dr. Maria Chasiotou contributed to the imaging analysis and explanations.

\section{Statement of Ethics}

The authors have no ethical conflicts to disclose.

\section{Disclosure Statement}

The authors have no proprietary interests, financial support, or any other conflicts of interest to report. 


\section{References}

1 Gerber B, Kiwanuka P, Dhariwal D: Orbital fractures in children: a review of outcomes. Br J Oral Maxillofac Surg 2013;51:789-793.

-2 Nolasco FP, Mathog RH: Medial orbital wall fractures: classification and clinical profile. Otolarygol Head Neck Surg 1995;112:549-556.

3 Boulos PR, Bernardino CR, Rubin PA: Pneumocele - a rare cause of air in the orbit. Am J Ophthalmol 2004;138:168-169.

4 Snell BJ, Flapper W, Moore M, et al: Management of isolated fractures of the medial orbital wall. J Craniofac Surg 2013;24:291-294.

5 Thiagarajah C, Kersten RC: Medial wall fracture: an update. Craniomaxillofac Trauma Reconstr 2009;2:135-139.

-6 Lee WT, Kim HK, Chung SM: Relationship between small-size medial orbital wall fracture and late enophthalmos. J Craniofacial Surg 2009;20:75-80.

7 Burm JS, Chung CH, Oh SJ: Pure orbital blowout fracture: new concepts and importance of medial orbital blowout fracture. Plast Reconstr Surg 1999;103:1839-1849.

8 Burnstine MA: Clinical recommendations for repair of orbital facial fractures. Curr Opin Ophthalmol 2003;14:236-240.

-9 Segrest DR, Dortzbach RK: Medial orbital wall fractures: complications and management. Ophthal Plast Reconstr Surg 1989;5:75-80.

10 Joseph JM, Glavas IP: Orbital fractures: a review. Clin Ophthalmol 2011;5:95-100.

11 Back CP, McLean NR, Anderson PJ, David DJ: The conservative management of facial fractures: indications and outcomes. J Plast Reconstr Aesthet Surg 2007;60:146-151.

-12 Chiu WC, Lih M, Huang TY, et al: Spontaneous orbital subcutaneous emphysema after sneezing. Am J Emerg Med 2008;26:381.e1-381.e2.

13 Rosh AJ, Sharma R: Orbital emphysema after nose blowing. J Emerg Med 2008;34:327-329.

14 Papadimitriou P, Ntomouchtsis A, Antoniades K: Delayed traumatic ocular emphysema: a case report. Oral Surg Oral Med Oral Pathol Oral Radiol Endod 2006;102:e18-e20.

15 Taguchi Y, Sakakibara Y, Uchida K, Kishi H: Orbital emphysema following nose blowing as a sequel of a snowboard related head injury. Br J Sports Med 2004;38:E28.

-16 Silver HS, Fucci MJ, Flanagan JC, Lowry LD: Severe orbital infection as a complication of orbital fracture. Arch Otolaryngol Head Neck Surg 1992;118:845-848. 


\section{Case Reports in Ophthalmology}


Fig. 1. Clinical appearance of our patient. Top row: at presentation, our patient had obvious difficulties to hold his right eyelid open in primary position due to ecchymosis, and there is a moderate limitation of upgaze. Middle row: one week after the trauma, there is still some limitation of upgaze, but the ecchymosis has resorbed enough so that he can hold the eyelid open. Bottom row: Three months after the injury, we did not observe any functional anomaly in primary or upward movement.

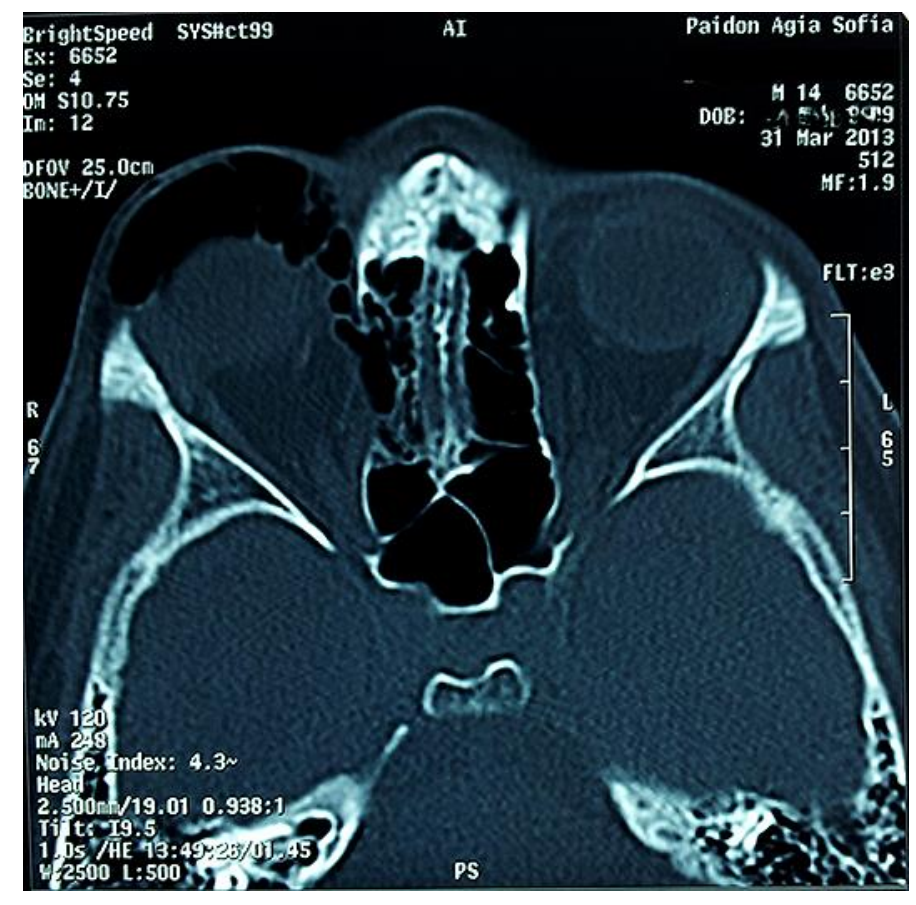

Fig. 2. Axial CT scan. Extensive subcutaneous presence of air in the right palpebra. 


\section{Case Reports in Ophthalmology}

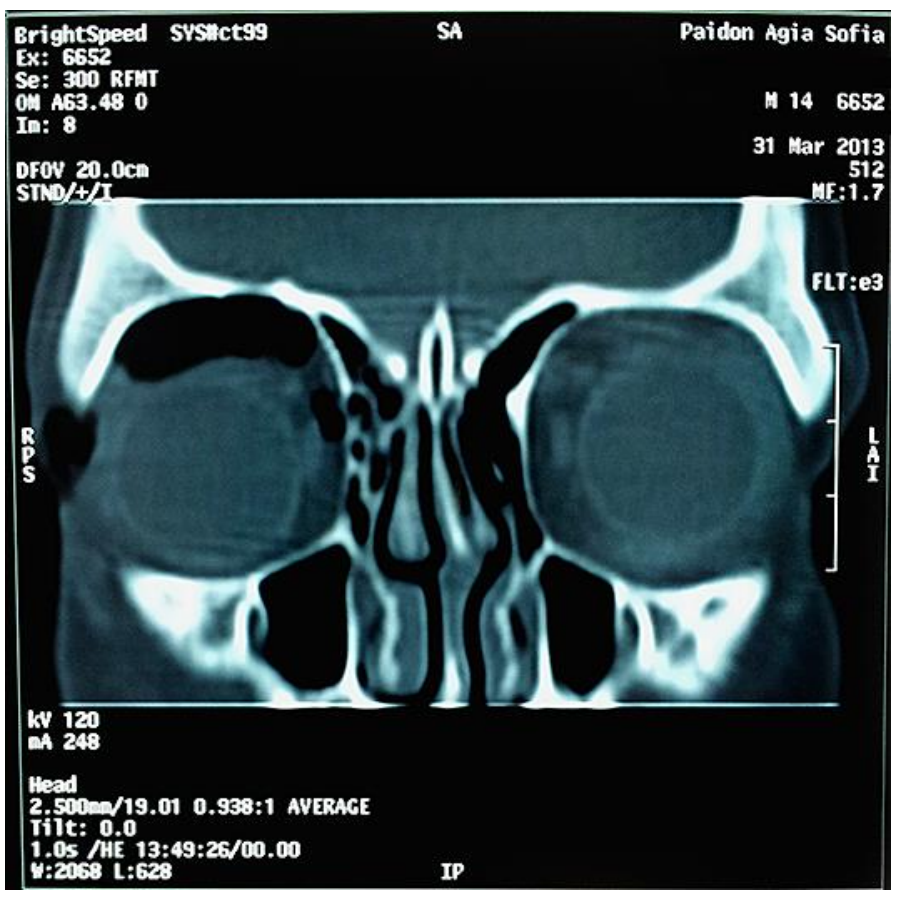

Fig. 3. Coronal CT scan. Disruption of the right lamina papyracea. Presence of air at the roof of the orbit.

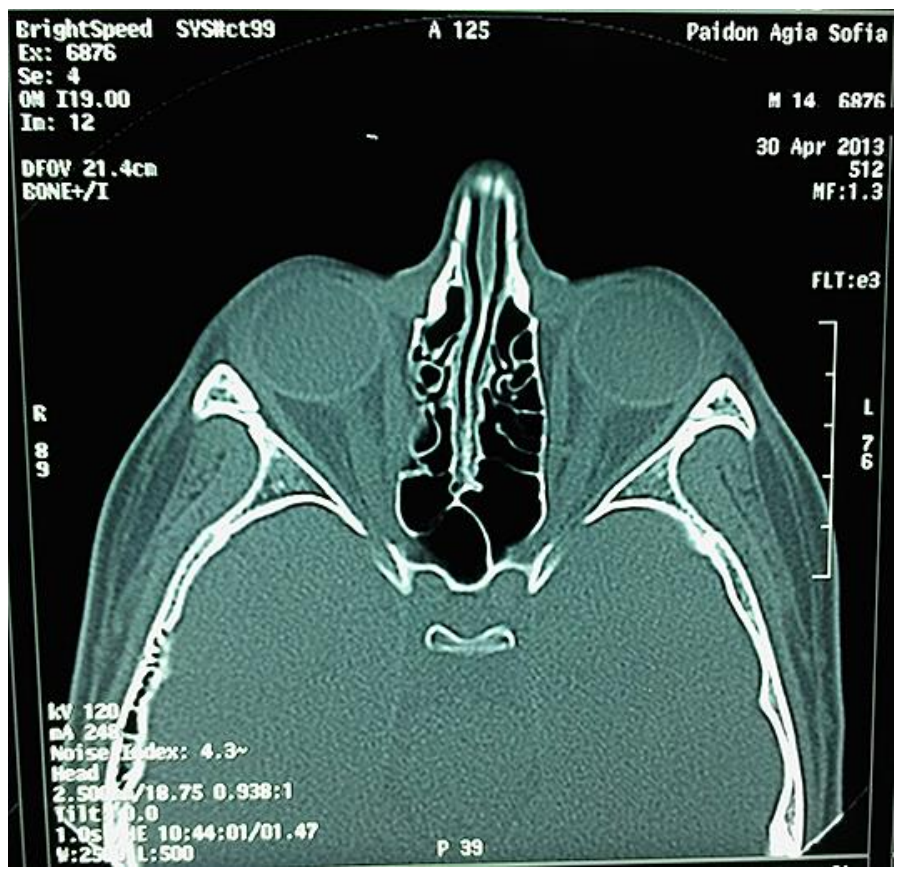

Fig. 4. Axial CT scan. One month after the trauma. Complete resolution of air in the right palpebra. 